\title{
The non-Boussinesq lock-exchange problem. Part 2. High-resolution simulations
}

\author{
By V. K. BIRMAN,,$^{1}$ J. E. MARTIN ${ }^{1,2}$ AND E. MEIBUR $\dagger^{+1}$ \\ ${ }^{1}$ Department of Mechanical and Environmental Engineering, University of California, \\ Santa Barbara, CA 93106, USA \\ ${ }^{2}$ Department of Mathematics, Christopher Newport University, Newport News, VA 23606, USA
}

(Received 26 March 2004 and in revised form 12 February 2005)

The present investigation explores the unsteady dynamics of large density contrast non-Boussinesq lock-exchange flows by means of high-resolution two-dimensional simulations of the incompressible variable-density Navier-Stokes equations, employing a combination of spectral and compact finite-difference methods. For small density contrasts, the simulations closely reproduce earlier Boussinesq results for corresponding flows. Across the entire range of density contrasts, good agreement is obtained between the computed front propagation velocities and corresponding experimental observations reported in Part 1 of this investigation and by other authors. The simulations yield the required quantitative information with respect to the light and dense front heights, their propagation velocities, and the spatial structure of the dissipation fields in order to determine conclusively which of the scenarios developed in Part 1 is observed in reality. Simulations are conducted for fluids with the same kinematic viscosity, as well as for fluids with the same dynamic viscosity. For both slip and no-slip boundary conditions, and for all $R e$ values, we find that for larger density contrasts, the dense front dissipates an increasing amount of energy. In contrast, the energy dissipated by the light front remains near its Boussinesq level for all values of the density ratio. In addition, for all density ratios, the height of the light front is very close to half the channel height, and it propagates with a non-dimensional velocity close to a half. This provides strong evidence that the dynamics of the light front is indeed approximated by the energy-conserving solution described in an earlier theoretical analysis. In contrast, the height of the dense front is substantially less than half the channel height. In addition, its velocity is close to the value derived in Part 1 for a dissipative gravity current. Together with the above results for the dissipation field, this confirms that the dense front behaves as a dissipative gravity current.

\section{Introduction}

In Part 1 of the present investigation (Lowe, Rottman \& Linden (2005) hereinafter referred to as LRL), a theoretical analysis of non-Boussinesq gravity currents in the lock-exchange configuration is developed. It attempts to explain the dynamics of the overall flow, which consists of a light front advancing along the top of the reservoir, and a dense front propagating along the bottom. A first scenario for the evolution of this flow in the presence of moderate density contrasts is developed based on the assumption that both fronts are energy conserving. For this case, the analysis

$\dagger$ Author to whom correspondence should be addressed. 
by Benjamin (1968) shows that the fronts will advance at different speeds, while their heights will be equal to half the channel width (cf. also Rottman \& Linden 2001). Volume conservation then demands that these energy conserving fronts are connected by an expansion wave, and that a bore forms between the dense front and the expansion wave (as discussed in $\S 3$ of LRL).

LRL analyse the expansion wave and the internal bore on the basis of the twolayer shallow-water equations, following the studies by Rottman \& Simpson (1983) and Keller \& Chyou (1991). Here, the difficulty arises that it is unknown what fraction of the dissipation associated with the internal bore occurs in each of the two layers. Past theoretical analyses had been developed for both limiting cases, i.e. all of the dissipation occurring either in the lower expanding layer (Yih \& Guha 1955; Chu \& Baddour 1977; Wood \& Simpson 1984) or in the upper, contracting layer (cf. Klemp, Rotunno \& Skamarock 1997). Since the spatial distribution of the dissipation is difficult to measure experimentally, LRL derive results for each of these two potential scenarios. They furthermore demonstrate that a third scenario is possible, in which the expansion wave is connected directly to a dissipative dense current head, without the formation of an internal bore. Hence, based on the above theoretical considerations there appear to be three plausible scenarios for the evolution of nonBoussinesq gravity currents in the lock-exchange configuration. They are distinguished by the front heights, their propagation velocities, and the spatial distribution of the dissipation field.

Information about the dissipation field is generally hard to obtain from experimental measurements. In addition, it is difficult to realize a wide range of density ratios with 'standard' fluids that can be handled easily in the laboratory. For this reason, the experiments of LRL are limited to density ratios above approximately 0.6. Gröbelbauer, Fanneløp \& Britter (1993) employ pairs of gases in order to extend the range of density ratios, but that approach has its own difficulties regarding the accurate determination of front heights and propagation velocities. Consequently, here we employ two-dimensional nonlinear Navier-Stokes simulations in order to obtain the information required to distinguish between the various plausible scenarios for non-Boussinesq gravity currents derived by LRL, as outlined above. The goal is to provide conclusive evidence as to which one of these scenarios will develop in reality.

As explained in the review of the pertinent literature by LRL, non-Boussinesq effects can be important in a number of safety and environmental problems, ranging from industrial processes involving gases of widely differing densities to fires in enclosed structures such as tunnels (Grant, Jagger \& Lea 1998; Kunsch 1999, 2002), and environmental phenomena such as snow avalanches. To date, most experimental, theoretical and computational studies have addressed Boussinesq situations. The relatively few investigations of gravity currents that have accounted for non-Boussinesq effects do so either on the basis of a box model approach (cf. Huppert 1998), or the thin-layer or shallow-water equations, such as in the work of Fanneløp \& Jacobsen (1984). Studies along these lines can be quite powerful in terms of providing information on the global properties of density-driven currents. On the other hand, they do not allow for a detailed investigation of the structure and dynamics of these currents. Specifically, they do not provide information regarding the spatial distribution of viscous dissipation rates. In this regard, highly resolved numerical simulations offer new opportunities, as demonstrated by the recent Boussinesq investigations of density-driven (Härtel et al. $2000 a, b$ ) and particle-driven (Necker et al. 2002, 2005) currents. Necker et al. (2002) compare two- and three-dimensional simulations for identical parameter combinations. A main difference concerns the two-dimensional spanwise vortices, which survive 


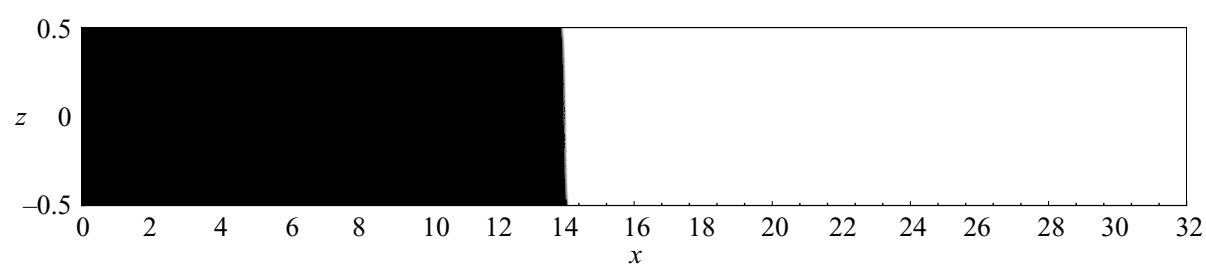

FiguRE 1. The classic lock-exchange configuration. A membrane initially divides the rectangular container of length $L$ and height $H$ into two compartments. The left-hand chamber is filled with fluid of density $\rho_{1}$, while the right-hand chamber contains fluid of smaller density $\rho_{2}$. Upon release of the membrane, a dense current moves rightward along the lower boundary, while the light fluid propagates leftward along the upper boundary.

over long times in two-dimensional simulations, whereas they are subject to more rapid breakdown in three-dimensional simulations, as a result of instabilities in the spanwise direction. These and other simulations have provided considerable insight into the dynamics and instabilities of the current fronts, and into the dissipation, mixing and sedimentation processes dominating them. The present investigation hence aims to deepen our understanding of non-Boussinesq gravity currents through highly resolved numerical simulations, and to obtain the quantitative information required to determine which of the potential scenarios for such currents will be seen in the real flow.

Section 2 presents the equations governing non-Boussinesq lock-exchange flows, along with the relevant non-dimensionalization process. Section 3 provides detailed information regarding the computational approach for integrating the governing equations. In $\S 4$, we analyse the results obtained from our current simulations, with respect to the heights and propagation speeds of the fronts, and the detailed energy budget of the flow. Furthermore, the effects of initial and boundary conditions are discussed, along with those of the Reynolds number. Section 5 summarizes the findings and draws the main conclusions.

\section{Basic equations}

The simulations employ a rectangular channel of height $H$ and length $L$, cf. figure 1 . The channel is filled with two miscible fluids initially separated by a membrane located at $x_{0}=14$. While the left compartment holds a fluid of density $\rho_{1}$, the right reservoir is filled with a fluid of lower density $\rho_{2}$. This initial configuration causes a discontinuity of the hydrostatic pressure across the membrane, which sets up a predominantly horizontal flow once the membrane is removed. The denser fluid moves rightward along the bottom of the channel, while the lighter fluid moves leftward along the top.

In most studies of gravity currents to date, the Boussinesq approximation has been employed, i.e. relative density variations were assumed to be small throughout the flow field. In the present study, our intention is to consider cases in which the density ratio of the dense and the light fluid may in fact be very large, so that the Boussinesq approximation cannot be invoked. Instead the full incompressible Navier-Stokes equations for variable density flows have to be solved. These read,

$$
\begin{aligned}
\nabla \cdot \boldsymbol{u} & =0, \\
\rho \frac{\mathrm{D} \boldsymbol{u}}{\mathrm{D} t} & =\rho \boldsymbol{g}-\nabla p+\nabla \cdot(2 \mu \mathbf{S}) .
\end{aligned}
$$


Here, $\mathrm{D} / \mathrm{D} t$ denotes the material derivative of a quantity, $\boldsymbol{u}=(u, v)^{T}$ indicates the velocity vector, $p$ the pressure, $\rho$ the density, and $\boldsymbol{S}$ the rate of strain tensor, while $\boldsymbol{g}=g \boldsymbol{e}_{g}$ represents the vector of acceleration due to gravity. In the following, we will keep the kinematic viscosity $v$ constant for both fluids. Later we will also consider the case of constant dynamic viscosity $\mu$. In deriving the above continuity equation, it is assumed that the material derivative of the density vanishes, i.e. $\mathrm{D} \rho / \mathrm{D} t=0$. This common assumption requires small diffusivities of the species concentration. The conservation of species is expressed by the convection-diffusion equation for the concentration $c$ of the heavier fluid. By assuming a density-concentration relationship of the form $\rho=\rho_{2}+c\left(\rho_{1}-\rho_{2}\right)$, we arrive at the following equation for the density field,

$$
\frac{\mathrm{D} \rho}{\mathrm{D} t}=K \nabla^{2} \rho,
$$

where the molecular diffusivity $K$ is taken to be constant. Note that the diffusive term must be kept in (2.3) in order to avoid the development of discontinuities in the computation of the density field. This holds true even if diffusive effects are very small, as in the case of liquids. In order to non-dimensionalize the above set of equations, the channel height $H$ is taken as the length scale, while the density $\rho_{1}$ of the heavier fluid serves as the characteristic density. Velocities are scaled by the buoyancy velocity $u_{b}=\sqrt{g^{\prime} H}$, in which $g^{\prime}$ denotes the reduced gravity (Simpson 1997), which is related to the dimensional acceleration due to gravity, $g$ by $g^{\prime}=g\left(\rho_{1}-\rho_{2}\right) / \rho_{1}=g(1-\gamma)$, where the density ratio is given by $\gamma=\rho_{2} / \rho_{1}<1$. A characteristic pressure $p$ is given by $u_{b}^{2} \rho_{1}$. We thus arrive at the following set of governing dimensionless equations

$$
\begin{aligned}
\nabla \cdot \boldsymbol{u} & =0, \\
\rho \frac{\mathrm{D} \boldsymbol{u}}{\mathrm{D} t} & =\frac{1}{1-\gamma} \rho \boldsymbol{e}_{g}-\nabla p+\frac{1}{R e} \nabla \cdot(2 \rho \boldsymbol{S}), \\
\frac{\mathrm{D} \rho}{\mathrm{D} t} & =\frac{1}{P e} \nabla^{2} \rho .
\end{aligned}
$$

If we were to make use of the Boussinesq assumption (2.5) would instead simplify to

$$
\frac{\mathrm{D} \boldsymbol{u}}{\mathrm{D} t}=\rho \boldsymbol{e}_{g}-\nabla p+\frac{1}{R e} \nabla^{2} \boldsymbol{u} .
$$

Note that we cannot obtain (2.7) from (2.5) merely by substituting $\gamma=1$, as the hydrostatic pressure field absorbed into the variable $p$ varies between the two cases.

In the present investigation, only horizontal channels are considered, so that $e_{g}$ is given by the unit vector $(0,0,-1)$. The three governing dimensionless parameters in (2.4)-(2.6) are the density ratio $\gamma$, the Reynolds number $R e$, and the Péclet number $P e$, respectively, which are defined as $R e=u_{b} H / v$ and $P e=u_{b} H / K$. They are related by the Schmidt number $S c=v / K$, so that $P e=R e S c$. It represents the ratio of kinematic viscosity to molecular diffusivity. For most pairs of gases, the Schmidt number lies within the narrow range between 0.2 and 5 . By means of test calculations we established that the influence of $S c$ variations in this range is quite small, so that in the simulations to be discussed below, we employ $S c=1$ throughout. It is to be kept in mind, however, that for liquids such as salt water, $S c \approx 700$.

For the purpose of numerical simulations, we recast (2.4)-(2.6) into the vorticitystreamfunction formulation. In this way, the incompressibility condition (2.4) is automatically satisfied throughout the flow field. Let $\psi$ be the streamfunction and $\omega$ the vorticity in the spanwise direction. Then the relations $\omega=\partial v / \partial x-\partial u / \partial z, u=\partial \psi / \partial y$, 
and $v=-\partial \psi / \partial z$ hold, and we obtain

$$
\begin{aligned}
\nabla^{2} \psi= & -\omega, \\
\frac{\mathrm{D} \omega}{\mathrm{D} t}= & \frac{1}{\operatorname{Re}} \nabla^{2} \omega-\frac{\rho_{x}}{(1-\gamma) \rho}+\frac{\rho_{z}}{\rho} \frac{\mathrm{D} u}{\mathrm{D} t}-\frac{\rho_{x}}{\rho} \frac{\mathrm{D} v}{\mathrm{D} t} \\
& +\frac{1}{\rho \operatorname{Re}}\left\{2 \rho_{x} \nabla^{2} v-2 \rho_{z} \nabla^{2} u+4 \rho_{x z} v_{z}+\left(u_{z}+v_{x}\right)\left(\rho_{x x}-\rho_{z z}\right)\right\} .
\end{aligned}
$$

If the dynamic viscosity $\mu$ is held constant instead of the kinematic viscosity $v,(2.9)$ takes the form

$$
\frac{\mathrm{D} \omega}{\mathrm{D} t}=\frac{1}{\rho \operatorname{Re}} \nabla^{2} \omega-\frac{\rho_{x}}{(1-\gamma) \rho}+\frac{\rho_{z}}{\rho} \frac{\mathrm{D} u}{\mathrm{D} t}-\frac{\rho_{x}}{\rho} \frac{\mathrm{D} v}{\mathrm{D} t} .
$$

We typically consider rectangular computational domains of length $L=32$. Initially, slip conditions are enforced along all of the walls. Consequently, we assign $\psi=0$ and $\omega=0$ along all boundaries. The concentration satisfies Neumann boundary conditions along all walls, in order to enforce zero diffusive mass flux. We will also evaluate the effect of no-slip conditions along the top and bottom boundaries in $\S 4.5$. These are implemented by taking $\psi=0$ along with $\omega=-\partial^{2} \psi / \partial z^{2}$.

\section{Computational approach}

The simulations employ equidistant grids in the rectangular computational domain. Spectral Galerkin methods are used in representing the streamwise dependence of the streamfunction and the vorticity fields

$$
\psi(x, z, t)=\sum_{l} \hat{\psi}_{l}(z, t) \sin (l \alpha x), \quad \omega(x, z, t)=\sum_{l} \hat{\omega}_{l}(z, t) \sin (l \alpha x),
$$

where $|l|<N_{1} / 2$ and $\alpha=2 \pi / L . N_{1}$ denotes the number of grid points in the streamwise direction. Vertical derivatives are approximated on the basis of the compact finitedifference stencils described by Lele (1992). As in the Boussinesq investigation of Härtel et al. (2000a), derivatives of the density field are computed from compact finite differences in both directions. At interior points, sixth-order spatially accurate stencils are used, with third- and fourth-order accurate stencils employed at the boundaries. The flow field is advanced in time by means of the third-order Runge-Kutta scheme described by Härtel et al. (2000a). The material derivatives of the velocity components appearing in the vorticity equation (2.9) are computed by first rewriting them in terms of the local time derivative plus the convective terms. The spatial derivatives appearing in the convective terms are then evaluated in the usual high-order way. The local time derivative is computed by backward extrapolation as follows,

$$
\left[\frac{\partial \boldsymbol{u}}{\partial t}\right]^{n}=\left(\boldsymbol{u}^{n}-\boldsymbol{u}^{n-1}\right) / \Delta t
$$

This approximation is consistently used during the successive Runge-Kutta substeps. Test calculations demonstrated that the low-order approximation of this term did not influence the results in a measurable way.

The Poisson equation for the streamfunction (2.8) is solved once per time step in Fourier space according to

$$
\left(\hat{\psi}_{l}^{m+1}\right)^{\prime \prime}-(l \alpha)^{2} \hat{\psi}_{l}^{m+1}=-\hat{\omega}_{l}^{m+1},
$$

with the prime denoting differentiation with respect to $z$. 


\subsection{Initial conditions}

In the present simulations, the flow field is initialized with the fluid at rest, i.e. $\boldsymbol{u}=0$ everywhere (corresponding to $\psi=0$ and $\omega=0$ ). The initial density field $\rho_{0}(x)$ could, in principle, be a step function with a density discontinuity at $x=x_{0}$. However, since the numerical scheme employs Fourier expansions in the $x$-direction, the density profile must be continuous, and a smooth transition between the initial densities in the left-hand and right-hand reservoirs has to be provided. Consequently, as the initial density field we specify an error function profile of the form,

$$
\rho_{0}(x)=\frac{\gamma+1}{2}-\frac{1-\gamma}{2} \operatorname{erf}(x \sqrt{\operatorname{ReSc}}) .
$$

This particular density profile was chosen because it satisfies the one-dimensional diffusion equation. The steepness of the profile (3.4) at the interface depends on the values of $R e$ and $S c$. Using the numerical approach described above, adequate resolution requires a grid size of $\Delta x \approx(R e S c)^{-1 / 2}$ in the streamwise direction (Härtel et al. $2000 a$ ), hence a grid of $4000 \times 200$ is typically employed. The simulations were performed on a 20-processor SGI machine, and the run times varied from 4 to 24 hours.

The results for $\gamma \approx 1$ should be nearly identical to those obtained in our earlier Boussinesq investigation (Härtel et al. 2000a). This fact will be used below in order to validate the computational code in the limit of density ratios near unity. For all other density ratios, the most meaningful validation of the numerical results involves a combination of monitoring the overall energy conservation and comparing computational results for the front propagation velocities with the experimental data of Gröbelbauer et al. (1993) and LRL.

\section{Results}

\subsection{Overview}

In order to validate the computational approach and the numerical simulation code, we first consider a case in which the initial density difference is very small. In this limiting situation, rather than solving the full variable-density Navier-Stokes equations, we could instead apply the Boussinesq approximation. Thus, the results obtained by solving the present variable-density equations for $\gamma \approx 1$ should agree with our earlier results based on the Boussinesq equations (cf. Härtel et al. 2000a). We begin by considering the case $\gamma=0.998$ for $R e=4000$. Figure 2 displays contours of the density field at several times, thereby demonstrating the temporal development of the interfacial region separating the two currents. Figure 2(a) shows that a sharp density gradient across the interface persists at time $t=2$. The heavier fluid has begun to move towards the right, and underneath the lighter fluid. At the same time, the lighter fluid is moving towards the left along the upper boundary, as it fills the space vacated by the denser fluid. This process is accompanied by the formation of two strong initial vortices near the heads of the fronts, and a shear layer in between, which separates the rightward-moving dense bottom current from the leftward-moving light top current. We shall use $x^{f}\left(x^{r}\right)$ to denote the time-dependent location of the foremost point of the dense (light) front, defined as the location where the density is equal to $(\gamma+1) / 2$. By means of interpolation, these front locations can be determined with a high degree of accuracy.

By time $t=10$ (cf. figure $2 b$ ), the symmetry with respect to the initial interface location at $x=14$ is maintained throughout the flow, as it should for the current with small density difference and the present set of initial and boundary conditions. The 

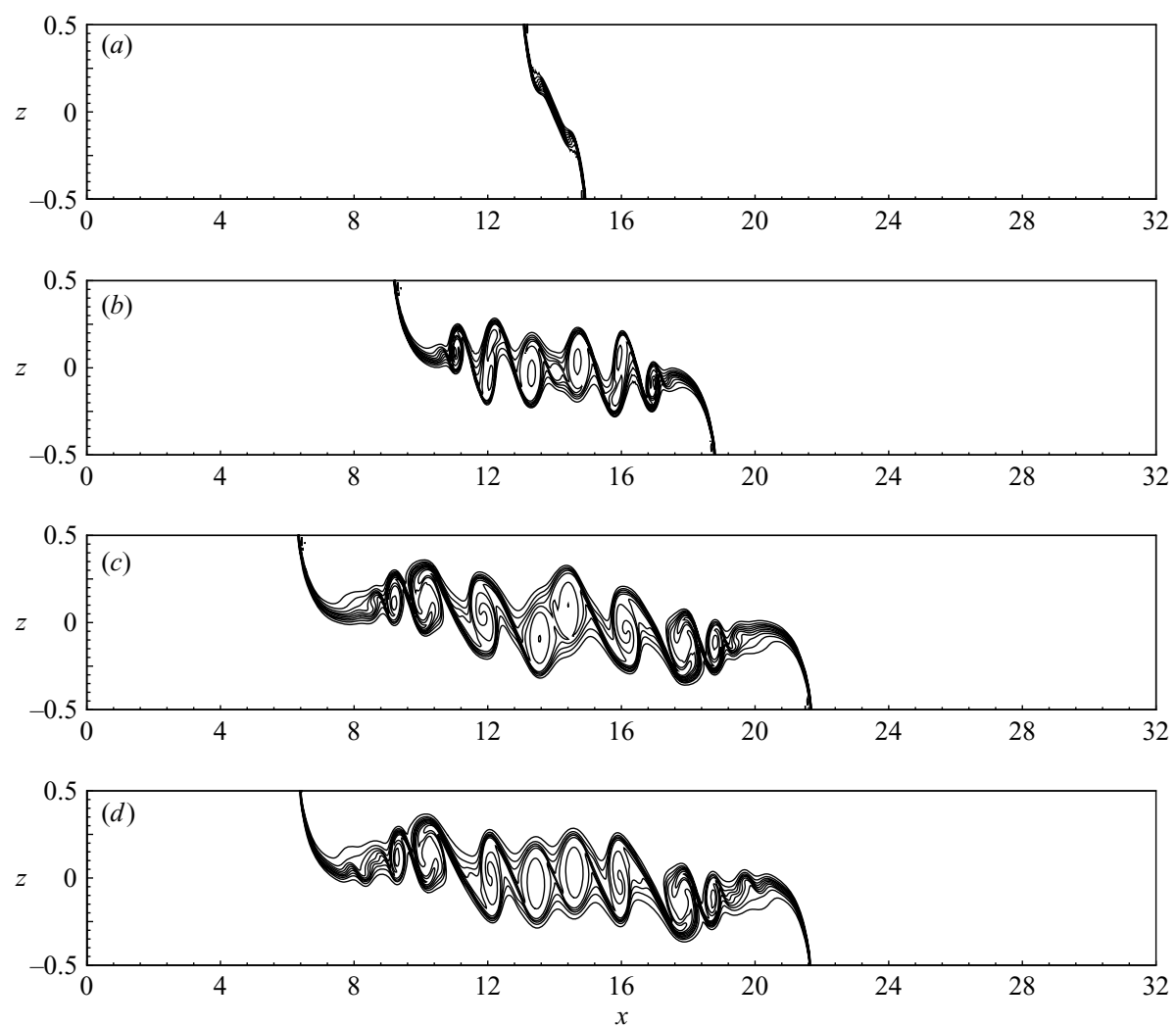

Figure 2. Concentration contours from a non-Boussinesq simulation for $\gamma=0.998$ and $R e=4000$, at times $(a) t=2,(b) 10$ and $(c)$ 16. (d) Concentration contours from a corresponding Boussinesq simulation at $t=16$. For this density ratios $\gamma=0.998$, the non-Boussinesq simulation is seen to give results that are very close to those of the Boussinesq simulation.

initial vortices now have increased in size and strength, and the Kelvin-Helmholtz instability emerging along the near horizontal sections of the interface is clearly visible. As was also observed in the earlier Boussinesq simulations of Härtel et al. (2000a), these Kelvin-Helmholtz billows may undergo a pairing process, similar to that observed in constant-density shear layers (Winant \& Browand 1974). The early stages of such a pairing process are observed for the two vortices closest to the initial interface location by $t=16$ (cf. figure $2 c$ ). Note that in a three-dimensional simulation, the Kelvin-Helmholtz billows tend to decay more rapidly, owing to instabilities in the spanwise direction (cf. Härtel et al. $2000 a$ and figure $2 a$ in LRL).

Figure $2(d)$ shows the density contours at the corresponding time from a simulation based on the Boussinesq approximation. By comparing figures $2(c)$ and $2(d)$, we see that for $\gamma \approx 1$, the present non-Boussinesq approach indeed closely reproduces the Boussinesq results. Similar agreement was also observed for other $R e$ values, which validates the present computational code in the limit of $\gamma \approx 1$.

As a next step, we consider $\gamma=0.92$, again for $R e=4000$. This density ratio is close to the limit where the Boussinesq approximation loses its validity. Figure 3(a) demonstrates that the point-symmetrical dynamics of the Boussinesq situation no longer develops. Even though initially the differences between the cases $\gamma=0.998$ and $\gamma=0.92$ are relatively small, asymmetries are seen to grow over time with respect 

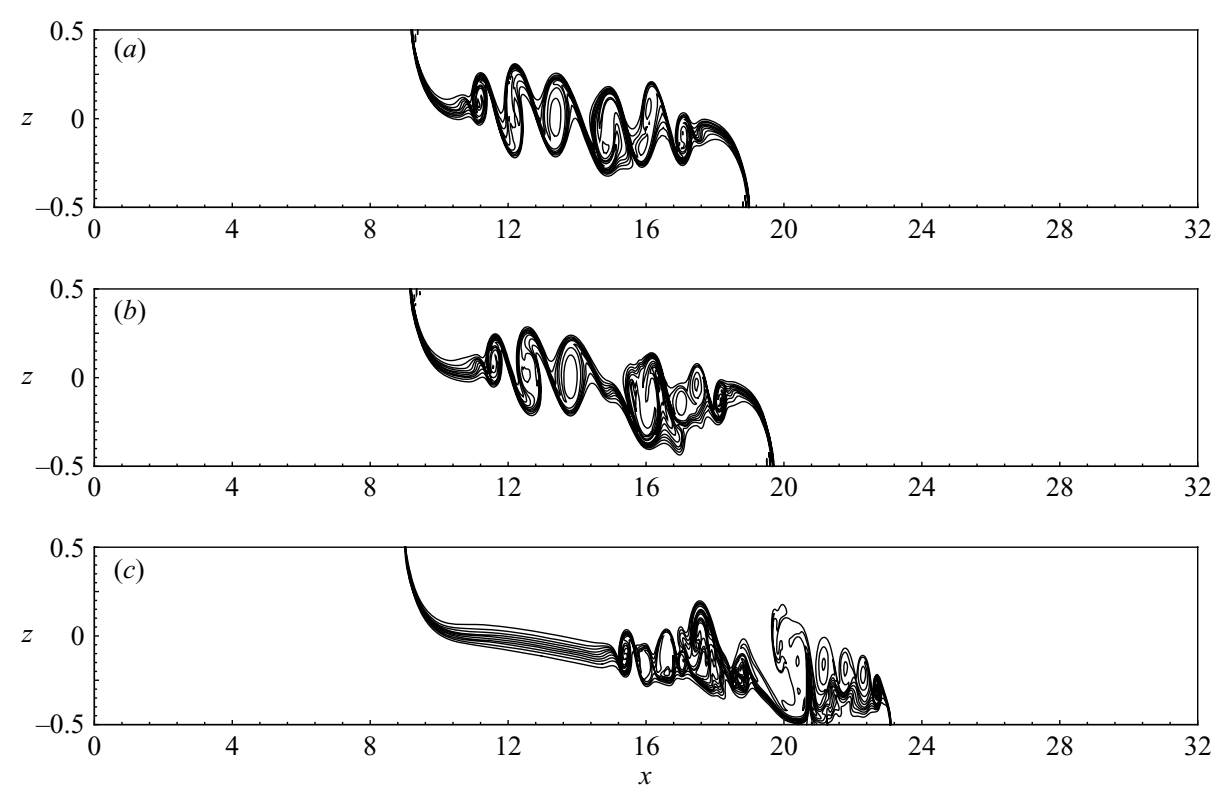

FIGURE 3. Concentration contours for $R e=4000$, at time $t=10$. (a) $\gamma=0.92$, (b) 0.7, 0.2. As the ratio of the densities decreases, asymmetries develop in the frontal velocities and the dynamics of the mixing layer vortices.

to the frontal locations and the mixing-layer vortices. While a pairing process with nearly perfect symmetry had been observed in the $\gamma=0.998$ simulation, this scenario no longer holds for $\gamma=0.92$.

Figure 3(b) depicts the flow for the relatively large density contrast of $\gamma=0.7$. Here the resulting asymmetry of the dominant flow features is much more pronounced. Already the initial vortex of the denser current is significantly stronger than that of the lighter current. The dense current propagates to the right along the bottom of the computational domain at a larger velocity than that of the light current propagating to the left along the top boundary, in agreement with the experiments by LRL (cf. their figure 3). Instabilities in the interfacial region evolve more rapidly near the rightward-moving dense front as compared to the receding light front.

For two fluids of vastly disparate densities, markedly different features emerge in the dynamics of the dense front (cf. figure $3 c$ ). Its height is considerably lower than before, and strong vortices form in close proximity to it. At the same time, the light front is now completely stabilized. In comparison to the case of $\gamma=0.7$, the dense front at time $t=10$ has propagated further, in good agreement with the experimental observations of Gröbelbauer et al. (1993). The expansion wave connecting the dense and light fronts is clearly visible. Note that the generation of vortical structures is limited to the region near the dense front. This lack of instabilities in the leftwardmoving light current is consistent with the linear stability analysis described in $\S 5$ of LRL. Across the dense current, the velocity difference, and hence the shear, is larger, which has a destabilizing effect.

The heights of the dense and light fronts represent important quantities in the analysis of the overall flow, as they will help determine which one of the proposed theoretical solutions is observed. In order to obtain quantitative data, we define the height $h(x)$ of the dense current by integrating the concentration field in the 

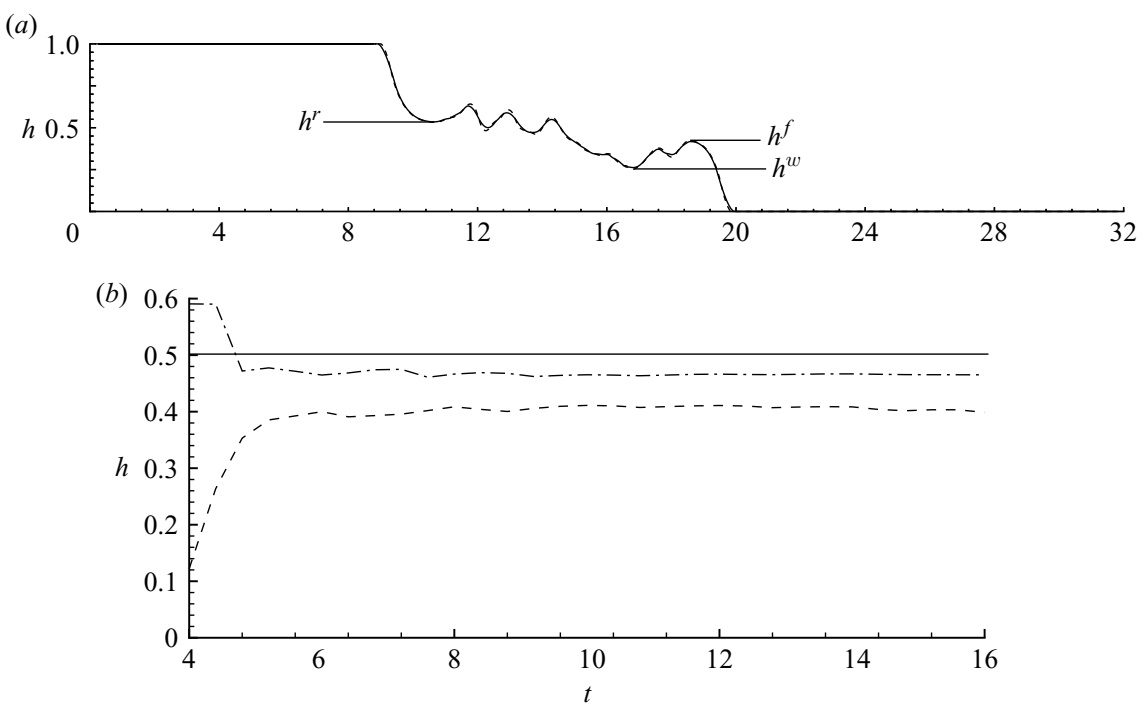

FiguRE 4. The height of the dense current for the flow depicted in $(b) .(a) h(x)$ evaluated by spatially averaging over $N=16$ (solid lines) and $N=10$ (dashed lines) grid points. The comparison indicates that the current height $h(x)$ is largely independent of $N$. The height at the end of the expansion wave $h^{w}$ is defined as the lowest point within the extended region of decreased height behind the dense front. The height $h^{f}$ of the dense front is the maximum of $h(x)$ between the end of the expansion wave and the tip $x^{f}$ of the dense front. The height of the light front $h^{r}$ is evaluated at the first minimum of $h(x)$, coming from the left-hand boundary. $(b)$ The height $h^{r}(t)$ of the light front (dash-dot line) remains close to the value of 0.5 for an energy conserving front (solid line) throughout the simulation. In contrast, the height $h^{f}(t)$ of the dense front (dashed line) is substantially below that value.

$z$-direction

$$
h^{*}(x)=\frac{1}{(1-\gamma)} \int_{0}^{1} \rho(x, z) \mathrm{d} z-\frac{\gamma}{(1-\gamma)},
$$

and subsequently taking a moving average of $h^{*}(x)$ over $N$ grid points in the $x$-direction, in order to eliminate high-frequency oscillations

$$
h\left(x_{i}\right)=\frac{1}{N+1} \sum_{j=-N / 2}^{N / 2} h^{*}\left(x_{i+j}\right) .
$$

The comparison shown in figure 4(a) for $N=10$ and 16 (for the flow shown in figure 4) indicates that the resulting measure of the current height is largely independent of $N$. Use of (4.1) removes the need to make a subjective judgement of the depth of the current, as has usually been the case in previous experiments. Furthermore, it accounts for the fact that the driving pressure for the current is given by the vertical integral of the density

$$
g^{\prime} h(x)=g \int_{0}^{H} \frac{\rho(x, z)-\rho_{1}}{\rho_{1}} \mathrm{~d} z .
$$

Consistent with the experiments of LRL, we observe a decrease in the height of the dense current behind the front. As indicated in figure 4, we take the lowest point in this region as the end of the expansion wave. Here, the height of the current is indicated by $h^{w}$. The highest value of $h$ downstream of this location is taken as the 

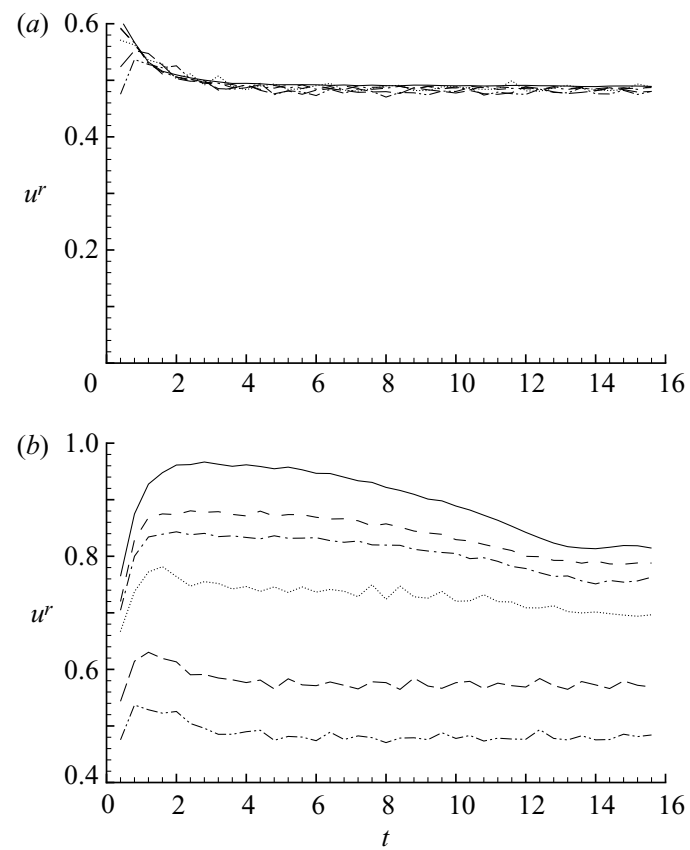

FIgURE 5. The $(a)$ light and $(b)$ dense current propagation velocities as functions of time for various density ratios. $\gamma=0.2$ (solid line), 0.26 (dashed line), 0.3 (dash-dot line), 0.4 (dotted line), 0.7 (long dash line), 0.998 (dash-dot-dot line). The velocity of the light front is seen to be independent of $\gamma$, while the dense front propagates more rapidly as the density contrast increases.

front height $h^{f}$. The height of the receding front is evaluated as $1-h^{r}$, where $h^{r}$ is the value at the location of the first minimum of $h(x)$, coming from the left (cf. figure 12 in LRL).

For the flow depicted in figure $3(b)$, figure $4(b)$ shows the front heights of the two currents as functions of time. After an initial transient, the light front height remains steady at a value of about 0.47 , which is close to the value of 0.5 for an energy conserving front. On the other hand, the height of the dense front is closer to 0.4, which is substantially below the value for an energy conserving front. This difference in heights provides a first indication that the two fronts evolve in fundamentally different ways.

To quantify the dense and light front speeds, respectively, we define $u_{f}=\mathrm{d} x^{f} / \mathrm{d} t$ and $u_{r}=\left|\mathrm{d} x^{r} / \mathrm{d} t\right|$. The dimensionless dense front speed $u_{f}$ is sometimes referred to as the Froude number of the dense current.

Figure 5 depicts the front speeds as functions of time for various values of $\gamma$. For all density ratios, we see the propagation velocity of the light front approaching a steady-state value just below 0.5 (cf. figure $5 a$ ). This front speed is in agreement with the value postulated by Benjamin (1968) for an energy-conserving current, cf. also LRL. In contrast, the propagation velocity of the dense front is seen to depend strongly on $\gamma$ (cf. figure $5 b$ ). As the density difference between the two fluids increases, the heavy front advances at a faster rate.

Figures 9 and 11 in LRL present a comparison of the present computed steadystate (or time-averaged, if no steady state exists) velocities for the light and dense fronts, respectively, with the data from the experiments of LRL, Gröbelbauer et al. 
(1993), and Keller \& Chyou (1991), as well as with the theoretical values proposed by LRL for an energy-conserving light front and a dissipative dense front. For the entire range of $\gamma$ values investigated here, the numerically computed front propagation velocities agree well with those observed in the experiments. The small remaining discrepancies are probably due to a combination of reasons. For example, the various combinations of gases and liquids employed in the experiments result in a range of experimental Reynolds and Schmidt numbers, whereas in the simulations those parameters are kept constant at $R e=4000$ and $S c=1$. In addition, the present values were obtained for slip boundary conditions. Furthermore, we must keep in mind that the simulations are strictly two-dimensional, and that three-dimensional effects might alter the front propagation velocity somewhat. Such slight variations between two- and three-dimensional front velocity values are observed, for example, in the Boussinesq simulations of particle-driven gravity currents by Necker et al. (2005). Finally, there may be imprecisions in the experimentally determined front velocity as well. Overall, the good level of agreement can be taken as further evidence for the validity of the present simulation data.

\subsection{Energy budgets}

Three different potential scenarios for the dynamics of the dense front in nonBoussinesq gravity currents were discussed in LRL. These scenarios are distinguished by the heights and the speeds of the fronts, by the existence of an internal bore, and by the ways in which the initially available potential energy is converted into kinetic energy, and subsequently dissipated into heat by viscous friction. It is difficult to measure the spatio-temporal evolution of these energetic quantities with high accuracy. Hence, in the following we will provide a computational analysis of the overall energy budget, in order to identify which one of the three potential solutions is most likely to be observed physically.

We will begin by commenting on the accuracy with which the overall energy is conserved in the numerical simulations. Then, we will discuss the temporal and spatial distribution of viscous dissipation, in order to establish whether or not it is predominantly localized in either the heavier or the lighter fluid. As discussed in LRL, past theoretical analyses of bores have been based on the assumption that all of the dissipation is localized either in the expanding layer (the lower layer in our case, Yih \& Guha 1955; Wood \& Simpson 1984), or in the contracting layer (cf. Klemp et al. 1997). Which one of these assumptions is closer to reality remains an unresolved issue. Lastly, we will contrast the amount of dissipation in the light front with that in the dense front. This comparison will demonstrate that it is indeed justified to consider the overall flow as a combination of a light energy-conserving front and a heavy dissipative front.

The equation for the time derivative of the kinetic energy can be obtained by multiplying the momentum equation (2.5) by $u_{i}$, which yields

$$
\frac{\mathrm{D}}{\mathrm{D} t}\left(\frac{1}{2} \rho u_{i} u_{i}\right)=-\frac{\partial}{\partial x_{i}}\left(p u_{i}\right)+\frac{1}{R e} \frac{\partial}{\partial x_{j}}\left(\rho s_{i j} u_{i}\right)-\frac{2}{R e} \rho s_{i j} s_{i j}-\frac{1}{1-\gamma} \rho v,
$$

where $\mathrm{D} / \mathrm{D} t$ indicates the material derivative and $s_{i j}$ denotes the rate-of-strain tensor $s_{i j}=\left(\partial u_{i} / \partial x_{j}+\partial u_{j} / \partial x_{i} / 2\right)$. Integration of (4.4) over the entire flow domain $\Omega$ provides an equation for the temporal evolution of the total kinetic energy $K$, which is the primary quantity of interest here

$$
\frac{\mathrm{d} K}{\mathrm{~d} t}=-\int_{\Omega} \frac{2}{R e} \rho s_{i j} s_{i j} \mathrm{~d} V-\frac{1}{1-\gamma} \int_{\Omega} \rho v \mathrm{~d} V, \quad K(t)=\int_{\Omega} \frac{1}{2} \rho u_{i} u_{i} \mathrm{~d} V .
$$


Note that the first two terms on the right-hand side of (4.4) are divergence terms which, in the present case, vanish after integration over $\Omega$ (see Necker et al. 2002).

The potential energy in the system is obtained from the integral

$$
E_{p}(t)=\int_{\Omega} \frac{1}{1-\gamma} \rho z \mathrm{~d} V
$$

and its time derivative is given by

$$
\frac{\mathrm{d} E_{p}}{\mathrm{~d} t}=\int_{\Omega} \frac{1}{1-\gamma} \frac{\mathrm{D}(\rho z)}{\mathrm{D} t} \mathrm{~d} V=\frac{1}{1-\gamma} \int_{\Omega} \rho v \mathrm{~d} V+\frac{1}{1-\gamma} \int_{\Omega} z \frac{\mathrm{D} \rho}{\mathrm{D} t} \mathrm{~d} V .
$$

The second term on the right-hand side of (4.7) can be rewritten by means of the transport equation (2.6). For simplicity, we neglect in the following the effects of diffusion in the concentration field on the potential energy (see Winters et al. 1995 for a discussion of such effects), and thus arrive at the following equation for $E_{p}$,

$$
\frac{\mathrm{d}}{\mathrm{d} t} E_{p}=\frac{1}{1-\gamma} \int_{\Omega} \rho v \mathrm{~d} V
$$

Summing (4.5) and (4.8), and taking into account that no change in potential energy occurs due to transport across domain boundaries, we find for $\mathrm{d} / \mathrm{d} t\left(K+E_{p}\right)$, i.e. for the change of total mechanical energy with time,

$$
\frac{\mathrm{d}}{\mathrm{d} t}\left(K+E_{p}\right)=-\frac{2}{R e} \int_{\Omega} \rho s_{i j} s_{i j} \mathrm{~d} V=-\int_{\Omega} \epsilon \mathrm{d} V=-\varepsilon,
$$

where $\epsilon$ represents the local rate of dissipation $\epsilon=(2 / R e) \rho s_{i j} s_{i j}$. Integrating (4.9) with respect to time yields $K+E_{p}+E_{d}=$ const. $=K_{0}+E_{p 0}$, with $E_{p 0}$ being the initial potential energy, $K_{0}$ the initial kinetic energy, and $E_{d}$ the time integral of the dissipation

$$
E_{d}(t)=\int_{0}^{t} \varepsilon(\tau) \mathrm{d} \tau
$$

By subtracting the potential energy of the light fluid,

$$
E_{a m b}=\frac{1}{1-\gamma} \int_{\Omega} \gamma z \mathrm{~d} V
$$

from the overall potential energy of the system, we obtain the available potential energy $E_{p 0}^{e}=E_{p 0}-E_{a m b}$, and $E_{p}^{e}=E_{p}-E_{a m b}$. Only this available potential energy can be converted into kinetic energy and, ultimately, be dissipated. Hence, we normalize all contributions to the overall energy budget with the initial available potential energy $E_{p}^{n}=E_{p}^{e} / E_{p 0}^{e}, K^{n}=K / E_{p 0}^{e}$, and $E_{d}^{n}=E_{d} / E_{p 0}^{e}$. Here, the superscript $n$ denotes normalized contributions. The time histories of these normalized kinetic energy, potential energy and dissipation values over the course of the simulations for $\gamma=0.998$ and 0.4 are shown in figure 6. It is obvious from these graphs that the overall energy is conserved to a high degree of accuracy during these simulations, even over fairly long times. The maximum error is of the order of $4 \%$, and can be attributed to the fact that we have neglected diffusive effects in the concentration field in the derivation of the energy equations. Furthermore, $E_{d}^{n}$ is evaluated with a comparatively low-order integration scheme.

\subsubsection{Spatial distribution of dissipation}

The local, instantaneous rate of dissipation $\epsilon$ is evaluated at each grid point as explained above. By weighting it with the dense fluid concentration $c=(\rho-\gamma) /(1-\gamma)$, we can associate a fraction of this rate of dissipation with the dense fluid ' 1 ', while the 

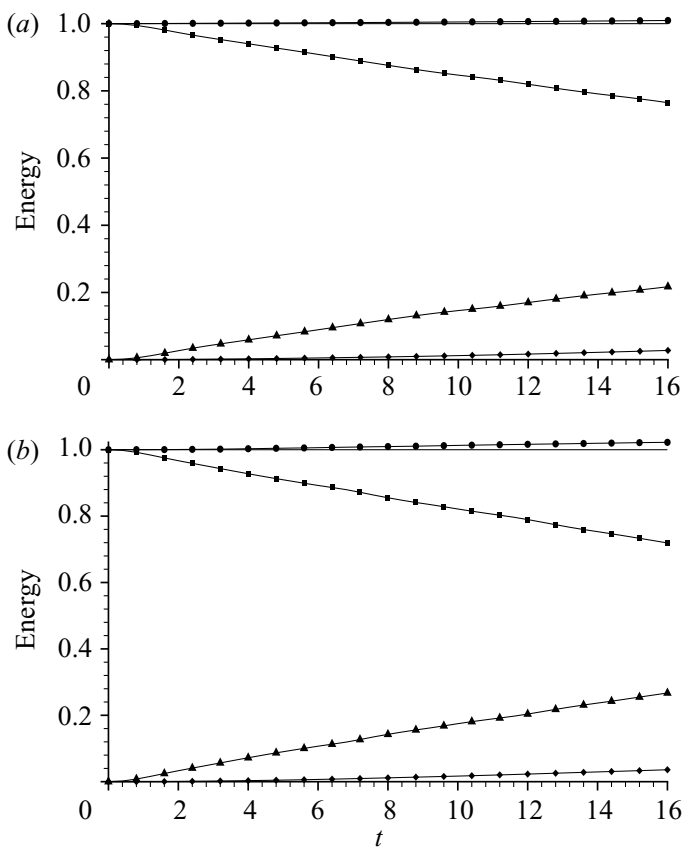

FiguRE 6. The normalized total energy (circles), kinetic energy (triangles), potential energy (squares) and dissipated energy (diamonds) as a function of time for $(a) \gamma=0.998$ and $(b) 0.4$. The solid line without symbols is drawn at a constant value of one. Overall energy is conserved to within about $1.5 \%$ in the simulations. With decreasing density ratios, the conversion of potential into kinetic energy proceeds at a slightly faster rate.

remainder is considered to occur within the light fluid ' 2 '. Thus, at each grid point, we obtain $\epsilon_{1}=c \epsilon$ and $\epsilon_{2}=(1-c) \epsilon$. Here, $\epsilon$ represents the entire local rate of dissipation, $\epsilon_{1}$ indicates the local rate of dissipation associated with the dense fluid, and $\epsilon_{2}$ is the local rate of dissipation within the light fluid. Figure 7(a) depicts the percentage of the overall dissipated energy that is associated with the dense fluid

$$
E_{d, 1}=\frac{1}{E_{d}} \int_{0}^{t} \int_{\Omega} \epsilon_{1}(\tau) \mathrm{d} V \mathrm{~d} \tau
$$

as a function of time for various density ratios. As expected, for the Boussinesq-like flow of $\gamma=0.998$, the dissipated energy is equally distributed between the dense and the light fluids. As the density ratio decreases, the lighter fluid dissipates slightly more energy than the heavier fluid. For $\gamma=0.7$, the percentage of the dissipated energy associated with the dense fluid has dropped to about $47 \%$. For the smallest density ratio that we simulated, $\gamma=0.2$, this percentage has decreased to $37 \%$. Figure $7(b)$ shows the percentage $E_{d, 1}$ at the final simulation time $t=16$ as a function of the density ratio. While $E_{d, 1}$ is seen to decay uniformly with $\gamma$, the figure suggests that even in the limit of very large density contrast this fraction will not go to zero.

While the above analysis focused on the relative fractions of the total dissipated energy associated with the dense and the light fluids, we will now analyse the fractions of the overall dissipated energy associated with the dense and light fronts. To this end, we assign all of the dissipation to the left (right) of $x_{0}$ to the light (dense) front $E_{d}^{f}=\int_{0}^{t} \int_{0}^{x_{0}} \int_{-0.5}^{0.5} \epsilon(\tau) \mathrm{d} z \mathrm{~d} x \mathrm{~d} \tau, E_{d}^{r}=\int_{0}^{t} \int_{x_{0}}^{L} \int_{-0.5}^{0.5} \epsilon(\tau) \mathrm{d} z \mathrm{~d} x \mathrm{~d} \tau, E_{d}=E_{d}^{f}+E_{d}^{r}$. 

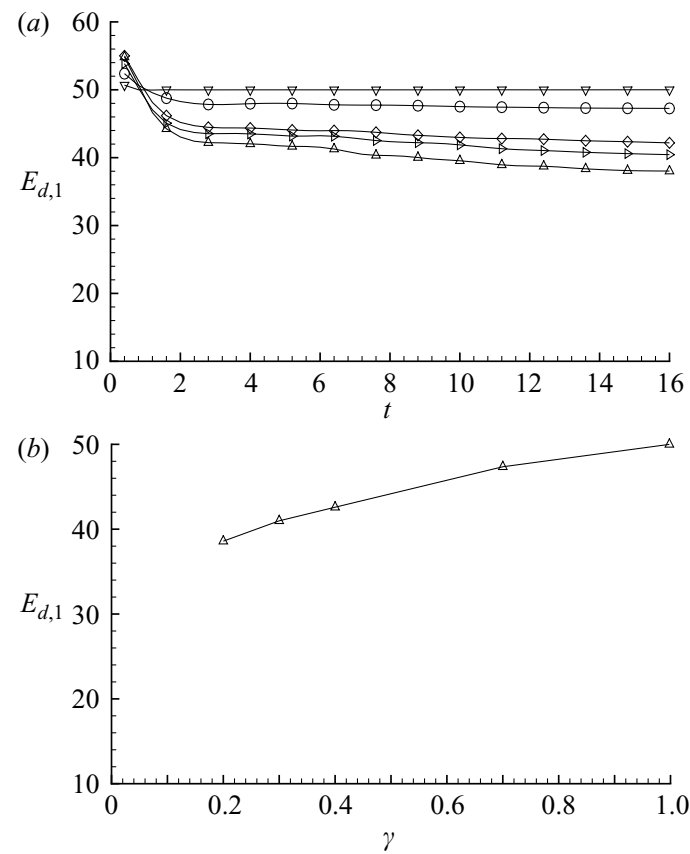

FIGURE 7. The percentage $E_{d, 1}$ of the overall dissipated energy associated with the dense fluid, as a function of $(a)$ time and $(b) \gamma$ at $t=16$. (a) Density ratios $\gamma=0.2$ (upward triangles), 0.3 (right triangles), 0.4 (diamonds), 0.7 (circles), and 0.998 (downward triangles). As the density ratio decreases, a larger fraction of energy is dissipated within the lighter fluid. After an initial transient, $E_{d, 1}$ remains approximately constant with time. (b) Shows as $\gamma$ decreases, a smaller fraction of the energy is dissipated within the denser fluid. However, this percentage does not tend to zero as the density contrast becomes very large.

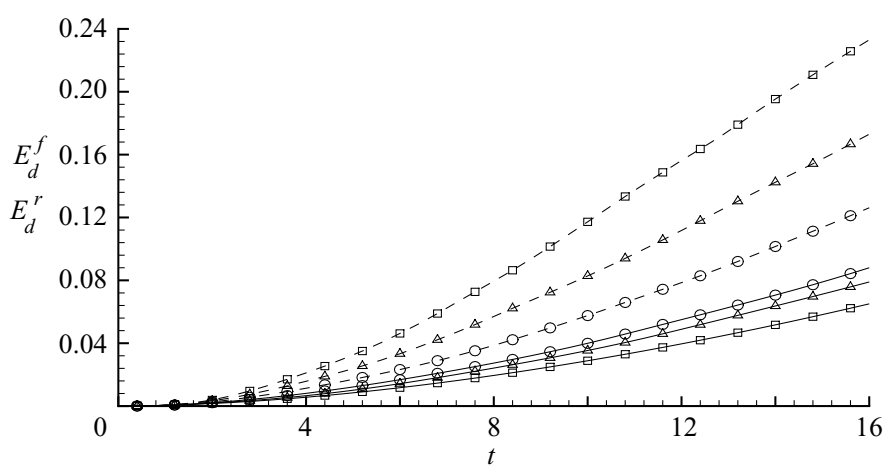

FIGURE 8 . The components of the overall dissipated energy associated with the dense front ( $E_{d}^{f}$, dashed lines) and the light front ( $E_{d}^{r}$, solid lines) as functions of time for the density ratios $\gamma=0.2$ (squares), 0.4 (triangles) and 0.7 (circles).

Figure 8 depicts $E_{d}^{r}$ and $E_{d}^{f}$ as functions of time for $\gamma=0.2,0.4$ and 0.7 . These results clearly show that, as the density contrast increases, the amount of energy dissipated within the light front decreases slightly from its Boussinesq value, whereas the dense front dissipates an increasing amount of energy. In combination with the computational data on the front heights and their propagation velocities, this provides convincing evidence for the argument put forward in LRL, namely that the 


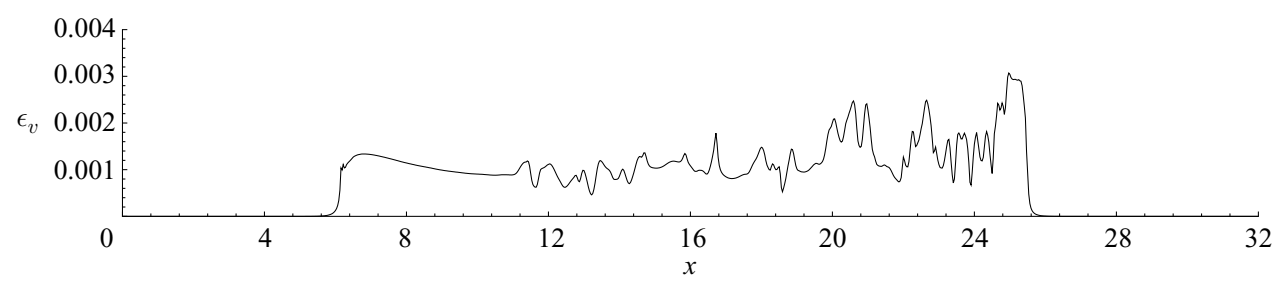

FiguRE 9. The vertically integrated dissipation rate $\epsilon_{v}(x)$ for $\gamma=0.4$ and $R e=4000$ at $t=16$ confirms that the level of dissipation is significantly higher in the dense front, as compared to the light front.
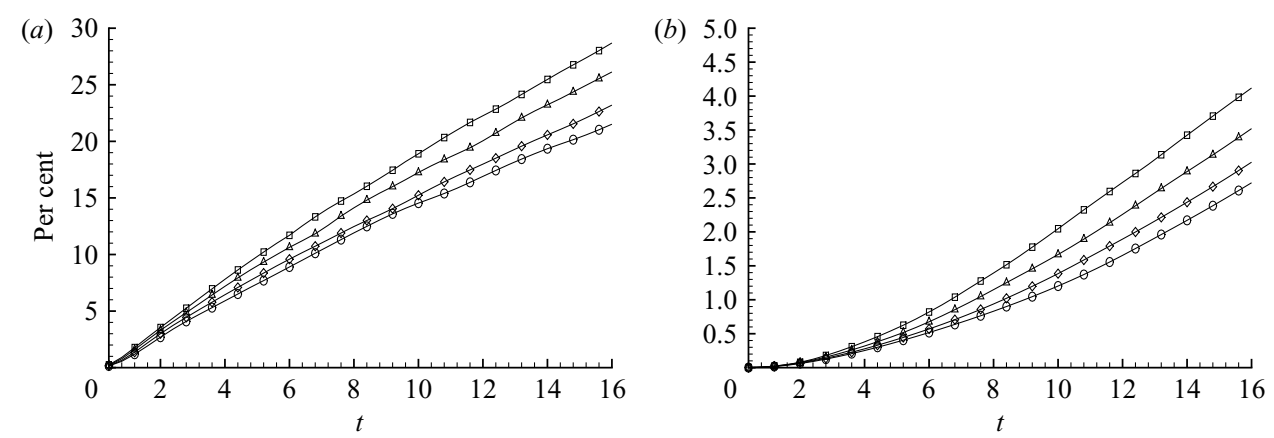

FiguRE 10. The fraction of the potential energy lost up to time $t$ that has been converted into (a) kinetic energy and $(b)$ dissipation, respectively, for various density ratios. $\gamma=0.2$ (squares), 0.4 (triangles), 0.7 (diamonds) and 0.998 (circles). For decreasing $\gamma$, dissipation accounts for a larger share of the lost potential energy, which is due to the increasingly dissipative nature of the dense front.

non-Boussinesq flow is composed of an essentially energy-preserving light front and a dissipative dense front, which are connected by an expansion wave. This is furthermore confirmed by figure 9, which shows the vertically integrated dissipation rate,

$$
\epsilon_{v}=\int_{-0.5}^{0.5} \epsilon \mathrm{d} z
$$

as a function of the streamwise coordinate. Clearly, the level of dissipation is significantly higher in the heavy front, as compared to the light front. Figure 10 shows what percentage of the potential energy lost up to time $t$ has been converted to kinetic energy, and what fraction has been dissipated, for various values of the density ratio. With decreasing $\gamma$, the dissipated energy accounts for a larger share of the lost potential energy. In light of figure 8 , we can conclude that this is due to the increasingly dissipative nature of the dense front.

In the following, we will discuss how the flow is influenced by the Reynolds number, as well as by the initial and boundary conditions.

\subsection{Influence of the Reynolds number}

The overall evolution of the dissipation field can be expected to vary with $R e$, owing to the changing balance of viscous and inertial forces. Hence, it is important to check the degree to which the above picture depends on $R e$. In order to illustrate the dependence of the overall flow on $R e$, we restrict our attention to the intermediate density ratio of $\gamma=0.4$. Figure 11 displays the concentration fields at time $t=10$ for the four $R e$-values of 1000, 4000 and 12000. As $R e$ increases, a larger number of 

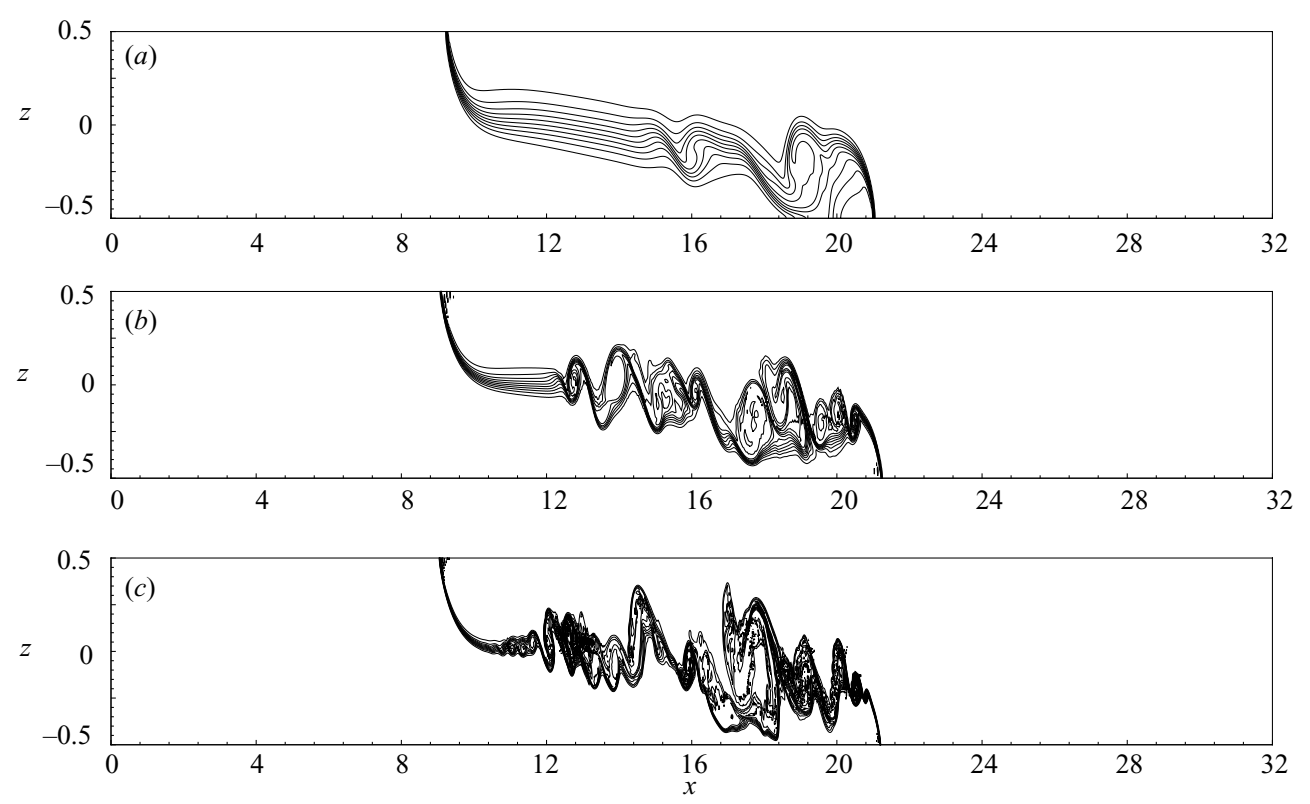

FIGURE 11. Concentration contours at $t=10$ for $\gamma=0.4$. (a) $R e=1000,(b) 4000$, (c) 12000. With increasing $R e$, a larger number of vortices appear in the region between the initial lock location and the dense front, while the light front maintains its essentially energy-conserving nature.

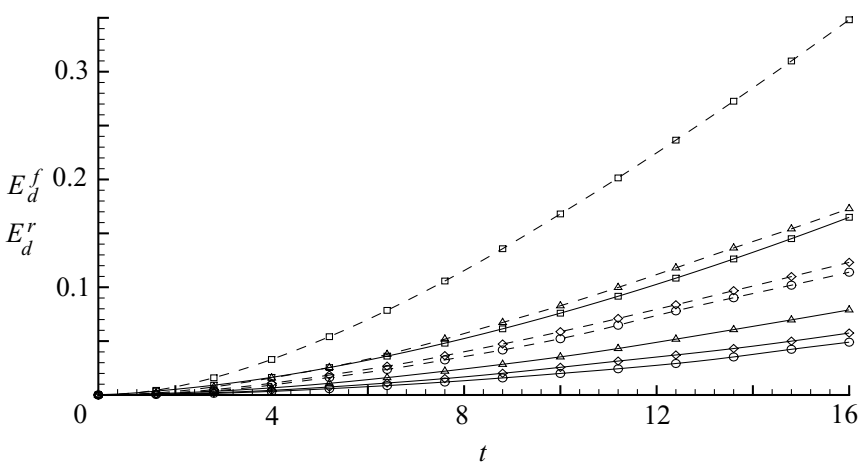

FIGURE 12. The components of the overall dissipated energy associated with the dense front ( $E_{d}^{f}$, dashed lines) and the light front ( $E_{d}^{r}$, solid lines) as functions of time for the density ratio $\gamma=0.4$ and $R e=1000$ (squares), 4000 (triangles), 8000 (diamonds), and 12000 (circles). The amount of energy dissipated in the light front is substantially lower than that in the dense front, for all values of $R e$.

vortical structures appear over a longer section of the interfacial region separating the two currents. However, even for the largest $R e$ values, the formation of these vortices is limited approximately to the dense front and the expansion wave, whereas strong vortices are not seen to form near the light front. Furthermore, the front velocities are seen to be essentially independent of $R e$ above $R e=4000$. This suggests that the light front remains essentially energy conserving across the entire $R e$ range.

Figure 12 compares the amounts of energy dissipated within each front as a function of time, for various values of Re. For all Reynolds numbers, the dense front dissipates 

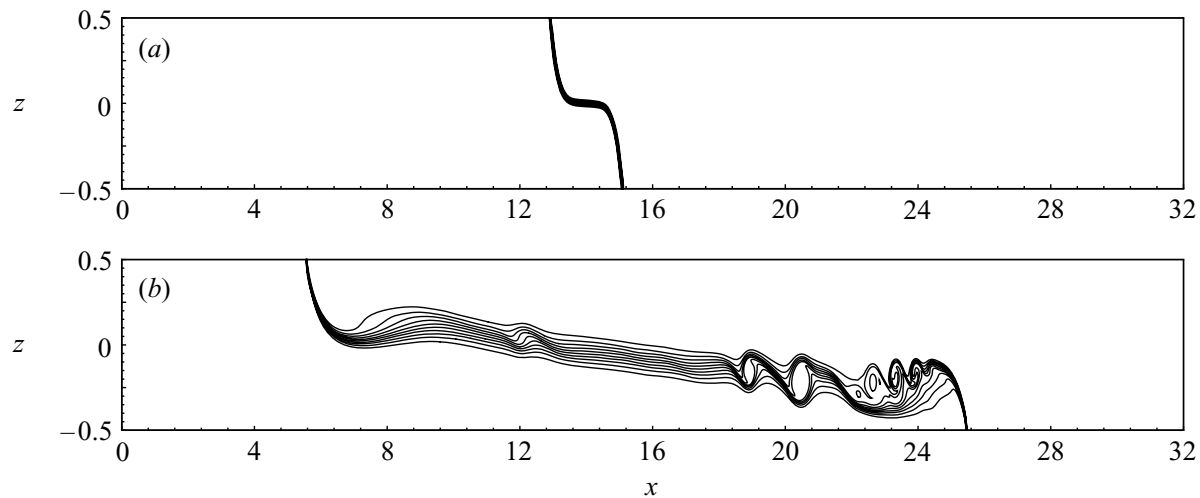

FiguRE 13. Influence of an initial staircase-like front geometry: Concentration contours for $\gamma=0.4$ and $R e=4000$ at times $t=0(a)$ and $16(b)$. Again, the flow evolves towards a stable, energy conserving light front connected to a dissipative dense front by an expansion wave.

substantially more energy than the light front, so that the distinction between the dissipative dense front and the more nearly energy conserving light front persists.

\subsection{Influence of the initial conditions}

In order to illustrate the effect of varying the initial geometry of the front, figure 13 shows concentration contours obtained for an initial 'staircase-like' profile. Here $\gamma=0.4$, with $R e=4000$. The early stages of the flow evolution clearly reflect the different initial geometry. Essentially each of the two steps within the staircase profile leads to the formation of separate light and heavy fronts. However, soon the light front emerging from the lower, right step combines with the dense front coming from the upper, left step, resulting in a long time evolution of the overall flow that is quite similar to the case of an initially vertical front. For long times, the front propagation velocities of the two cases are nearly identical. Again we observe a stable, energy conserving light front that is connected by an expansion wave to a dissipative dense front.

\subsection{Influence of the boundary conditions}

All of the simulations presented up to now employed slip boundary conditions along the horizontal walls. On the other hand, the work by Necker et al. (2005) for Boussinesq currents had shown that a significant amount of dissipation takes place in the viscous boundary layers near the solid walls which form as a result of the no-slip condition. Hence it is essential to check if the above picture of a dissipative dense front and a nearly energy conserving light front is modified in any significant way by the presence of a boundary layer. In order to illustrate the effects of imposing no-slip boundary conditions, figure 14 shows corresponding contour plots for $\gamma=0.4$ and $R e=4000$. This figure is to be compared with the slip flow of figure 3(b). It can be seen that the no-slip boundary slows both of the fronts down, cf. also figure 15, as had also been observed in the Boussinesq investigation of Härtel et al. (2000a). However, it does not change the dominant qualitative features of the flow, i.e., a stably propagating light front connected to a dissipative dense front characterized by the continuous formation of vortical structures.

With respect to the distribution of the dissipated kinetic energy, the no-slip flow is seen to behave very similarly to the slip flow. Hence even for no-slip flows, the 


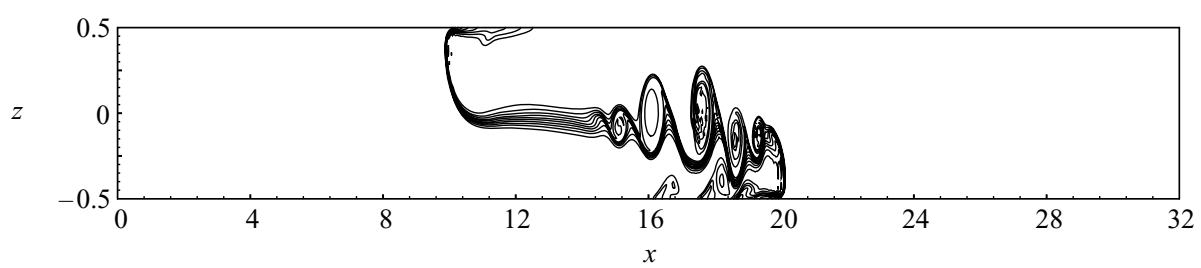

FIGURE 14. Concentration contours at $t=10$ for $\gamma=0.4$ and $R e=4000$, for no-slip boundary conditions along the horizontal walls. Similarly to the corresponding slip flow (shown in figure 3), the no-slip flow is characterized by intense vortical structures near the dense front, and a stable interface near the light front.

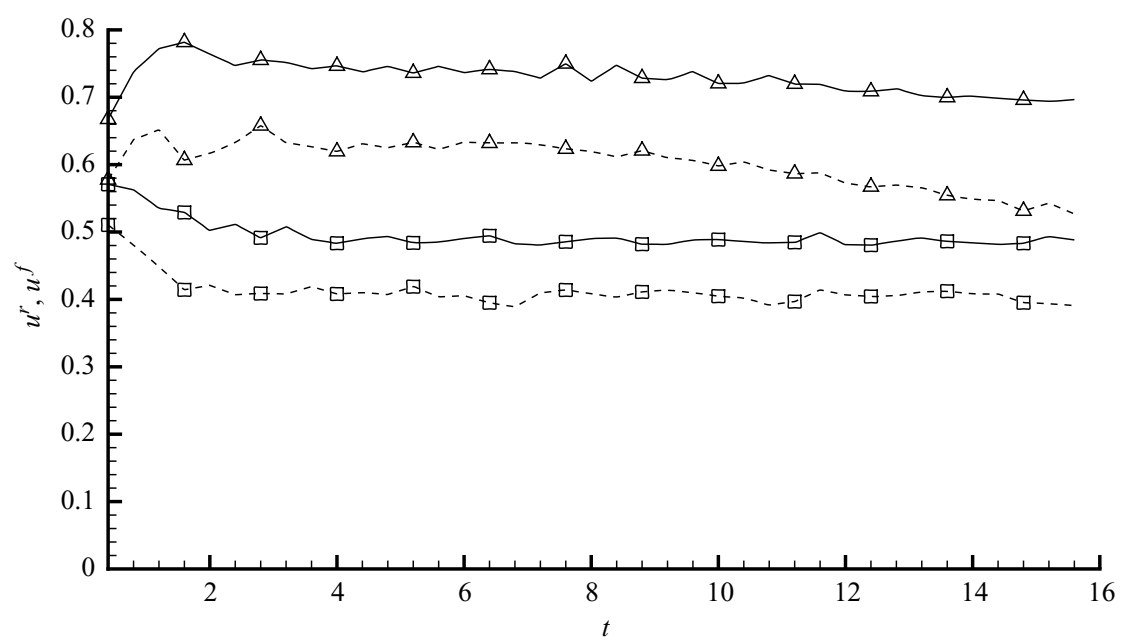

FIGURE 15. A comparison of dense (triangles) and light (squares) front velocities for $\gamma=0.4$ and $R e=4000$. The solid (dashed) lines refer to the case of slip (no-slip) boundary conditions along the horizontal walls. While both fronts are slowed down by the no-slip boundary condition, the dense front remains significantly faster than the light front.

concept of a nearly energy conserving light front joined by an expansion wave to a dissipative dense front remains valid.

\subsection{Simulations with constant dynamic viscosity $\mu$}

The vorticity transport equation (2.9) will simplify to (2.10) if we assume $\mu$ to be constant instead of $v$. For the Boussinesq case, these two situations are equivalent, but for larger density contrasts some differences emerge. For constant dynamic viscosity the formation of vortices is found to be limited to a smaller region close to the dense front. However, all of the above observations regarding the qualitative dependence of the front heights and velocities on $\gamma$ remain valid qualitatively, and to a good degree also quantitatively. The same holds for the spatial distribution of dissipation between the light and the heavy fluid, and the dense and the light front.

\section{Summary and conclusions}

The present investigation explores the unsteady dynamics of non-Boussinesq lockexchange flows by means of high-resolution two-dimensional simulations of the 
incompressible variable-density Navier-Stokes equations. These simulations yield the required quantitative information with respect to front heights, propagation velocities and dissipation fields in order to determine conclusively which of the three scenarios developed in LRL of the investigation is observed in reality. For both slip and noslip boundary conditions, and for all $R e$ values, we found that, for larger density contrasts, the dense front dissipates an increasing amount of energy. In contrast, the energy dissipated by the light front remains near its Boussinesq level for all values of the density ratio. In addition, the height of the light front is very close to half the channel height, and it propagates with a non-dimensional velocity very close to a half. This provides strong evidence that the dynamics of the light front is indeed closely approximated by the energy conserving solution described in the theoretical analysis of Benjamin (1968). In contrast, the height of the dense front is substantially less than half the channel height. In addition, its velocity is close to the value derived by LRL for a dissipative gravity current, and dissipation occurs primarily in the light fluid near the dense front. Together with the above results for the dissipation field, this confirms that the dense front behaves as a dissipative gravity current.

The very fruitful collaboration with Professors Paul Linden and Jim Rottman is gratefully acknowledged. This work has been supported by the National Science Foundation, both directly through grant CTS-0209194 as well as through an equipment grant. It was begun while J. E. M. was on sabbatical leave at UCSB. Additional support was received from the National Aeronautics and Space Administration under NASA Contract NAS1-97046, while J. E. M. was in residence at the Institute for Computer Applications in Science and Engineering at NASA Langley Research Center.

\section{REFERENCES}

Benjamin, T. B. 1968 Gravity currents and related phenomena. J. Fluid Mech. 31, 209.

Chu, V. H. \& Baddour, R. E. 1977 Surges, waves and mixing in two layer density stratified flow. Proc. 17th Congr. Intl Assocn Hydraul. Res. vol. 1, pp. 303-310.

FANNELøP, T. K. \& JACOBSEN, Ø. 1984 Gravitational spreading of heavy gas clouds instantaneously released. Z. Angew. Math. Phys. 35, 559.

Grant, G. B., Jagger, S. F. \& Lea, C. J. 1998 Fires in tunnels. Phil. Trans. R. Soc. Lond. A 356, 2873.

Gröbelbauer, H. P., Fanneløp, T. K. \& Britter, R. E. 1993 The propagation of intrusion fronts of high density ratio. J. Fluid Mech. 250, 669.

Härtel, C., Carlsson, F. \& Thunblom, M. 2000 $b$ Analysis and direct numerical simulation of the flow at a gravity-current head. Part 2. The lobe-and-cleft instability. J. Fluid Mech. 418, 213.

Härtel, C., Meiburg, E. \& Necker, F. 1999 Vorticity dynamics during the start-up phase of gravity currents. Il Nuovo Cimento C 22, 823.

Härtel, C., Meiburg, E. \& Necker, F. 2000a Analysis and direct numerical simulation of the flow at a gravity-current head. Part 1. Flow topology and front speed for slip and no-slip boundaries. J. Fluid Mech. 418, 189.

Huppert, H. E. 1998 Quantitative modelling of granular suspension flows. Phil. Trans. R. Soc. Lond. A 356, 2471.

Keller, J. J. \& Chyou, Y.-P. 1991 On the hydraulic lock-exchange problem. Z. Angew. Math. Phys. 42, 874 .

Klemp, J. B., Rotunno, R. \& SkAmarock, W. C. 1997 On the propagation of internal bores. J. Fluid Mech. 331, 81.

KunsCH, J. P. 1999 Critical velocity and range of a fire-gas plume in a ventilated tunnel. Atmos. Environ. 33, 13.

Kunsch, J. P. 2002 Simple model for control of fire gases in a ventilated tunnel. Fire Safety J. 37, 67. 
Lele, S. K. 1992 Compact finite difference schemes with spectral-like resolution. J. Comput. Phys. $103,16$.

Lowe, R. J., Rottman, J. W. \& Linden, P. F. 2005 The non-Boussinesq lock-exchange problem. Part 1. Theory and experiments. J. Fluid Mech. 537, 101-124.

Necker, F., Härtel, C., Kleiser, L. \& Meiburg, E. 2002 High-resolution simulations of particledriven gravity currents. Intl J. Multiphase Flow 28, 279-300.

Necker, F., Härtel, C., Kleiser, L. \& Meiburg, E. 2005 Mixing and dissipation in particle-driven gravity currents. J. Fluid Mech. (in press).

Rottman, J. W. \& Linden, P. F. 2001 Gravity currents. Stratified Flows in the Environment Grimshaw (ed. R. H. J. Kluwer), chap. 4.

Rottman, J. W. \& Simpson, J. E. 1983 Gravity currents produced by instantaneous releases of a heavy fluid in a rectangular channel. J. Fluid Mech. 135, 95.

Simpson, J. E. 1997 Gravity Currents in the Environment and the Laboratory. Cambridge University Press.

Winant, C. D. \& Browand, F. K. 1974 Vortex pairing: the mechanism of turbulent mixing-layer growth at moderate Reynolds number. J. Fluid Mech. 63, 237.

Winters, K. B., Lombard, P. N., Riley, J. J. \& D’Asaro, E. A. 1995 Available potential energy and mixing in density-stratified fluids. J. Fluid Mech. 418, 115-128.

Wood, I. R. \& Simpson, J. E. 1984 Jumps in layered miscible fluids. J. Fluid Mech. 140, 342.

YIH, C. S. \& GuHA, C. R. 1955 Hydraulic jump in a fluid system of two layers. Tellus 7, 358. 